\title{
The effect of ageing on shear wave elastography muscle stiffness in adults
}

\author{
Abdulrahman M. Alfuraih ${ }^{1,2,3}$ (D) Ai Lyn Tan ${ }^{2,3}$ (D) $\cdot$ Philip O'Connor ${ }^{3} \cdot$ Paul Emery ${ }^{2,3} \cdot$ Richard J. Wakefield $^{2,3}$ (1)
}

Received: 5 December 2018 / Accepted: 19 January 2019 / Published online: 14 February 2019

(c) The Author(s) 2019

\begin{abstract}
Background Skeletal muscle undergoes structural changes with ageing which may alter its biomechanical properties. Shear wave elastography (SWE) may detect these changes by measuring muscle stiffness.

Aims To investigate muscle stiffness in healthy young, middle-aged and elderly cohorts using SWE and correlate it with muscle strength and mass.

Methods Shear wave velocity (SWV) was measured in the quadriceps, hamstrings and biceps brachii of 26 young (range 20-35 years), 21 middle-aged (40-55) and 30 elderly (77-94) volunteers. The participants performed several muscle tests to evaluate their strength. The One-way ANOVA was used to test the muscle stiffness differences between the groups and the Pearson's correlation coefficient to evaluate the relationship between SWV and muscle strength.

Results The overall resting muscle SWV gradually decreased with age but was only significantly reduced in the elderly group $(p<0.001)$; with the exception of the vastus lateralis SWV where a significant difference was noted $(p<0.05)$ between young $(1.77 \mathrm{~m} / \mathrm{s})$, middle-aged $(1.64 \mathrm{~m} / \mathrm{s})$ and elderly $(1.48 \mathrm{~m} / \mathrm{s})$. The elderly group had on average $16.5 \%$ lower muscle stiffness compared to the young. SWV significantly correlated with muscle mass $(r=0.316)$, walking time $(r=-0.560)$, number of chair stands $(r=0.522)$, handgrip strength $(r=0.436)$ and isokinetic knee strength $(r=0.640)$. Sex and BMI did not explain any significant variation in SWV.

Conclusions Ageing was associated with a decline in skeletal muscle stiffness which positively correlates with muscle weakness. Further research is needed to evaluate the promising role of SWE as a biomarker for sarcopenia assessment and potential falls risk prediction in elderly individuals.
\end{abstract}

Keywords Elastography $\cdot$ Muscle $\cdot$ Stiffness $\cdot$ Age $\cdot$ Ageing $\cdot$ Ultrasound

Electronic supplementary material The online version of this article (https://doi.org/10.1007/s40520-019-01139-0) contains supplementary material, which is available to authorized users.

Abdulrahman M. Alfuraih

a.alfuraih@psau.edu.sa

1 Radiology and Medical Imaging Department, College of Applied Medical Sciences, Prince Sattam bin Abdulaziz University, Kharj, Saudi Arabia

2 Chapel Allerton Hospital, Leeds Institute of Rheumatic and Musculoskeletal Medicine, University of Leeds, Leeds, UK

3 NIHR Leeds Biomedical Research Centre, Leeds Teaching Hospitals NHS Trust, Leeds, UK

\section{Introduction}

It is known that muscle mass decreases with age [1]. Loss of mass and strength in elderly individuals are associated with increased risk of falls and loss of independence [2]. Histologically, ageing is associated with changes in muscle composition including myosteatosis, myofibrosis [3] and dysfunction in extracellular elastic fibres [4]. Such changes may alter the biomechanical properties resulting in changes to muscle stiffness.

Ultrasound shear wave elastography (SWE) is a new quantitative and non-invasive method used for assessing tissue stiffness. It has been widely utilised in breast, liver and thyroid [5]. However, there is minimal data on normative values for muscle stiffness and how it changes with age. SWE may offer new insights into how the ageing phenomenon affects the biomechanical properties of muscle and how 
it relates to muscle quality aspects. Previous evidence has demonstrated how tendon stiffness is significantly reduced in older populations [6]. However, no research to date has been dedicated to investigate how ageing may influence muscle stiffness using SWE and how it correlates with other primary variables such as muscle mass and strength.

Conventional biomechanical testing on animal models shows that muscle stiffness is not affected by ageing [7]. However, there are several, albeit not consistent, theoretical reasons why muscle stiffness might change with ageing. For example, it has been reported that an increase in collagen fibres in ageing muscle promotes an increase in muscle stiffness [8]. In contrast, others suggest that the elastic fibre system in the muscle extracellular matrix loses some of its resistance property and becomes softer with ageing [9]. Additional factors known to occur in aged muscles such as myosteatosis, variability in fibre sizes and impaired connective tissue support the second hypothesis [10,11].

The primary aim was to investigate to what extent muscle stiffness measured by SWE differed amongst healthy young, middle-aged and elderly cohorts. The secondary aim was to understand how muscle stiffness correlates with muscle strength and mass in different age groups.

\section{Methods}

\section{Study design}

This study was conducted in a cross-sectional design by recruiting healthy participants from three age groups, young (20-35 years), middle-aged ( $40-55$ years) and elderly (above 75 years), and testing their muscle stiffness, strength, function and body composition in a single visit. Assessments were conducted at the Leeds Biomedical Research Centre based at Chapel Allerton Hospital in Leeds, UK from May 2017 to September 2018.

\section{Eligibility and recruitment}

Participants' eligibility for the young and middle-aged groups was based on the following:(1) aged 18-35 years old or 40-55 years old, respectively; (2) being asymptomatic; (3) no previous history of musculoskeletal or neurological disorders; (4) not currently taking or previously taken a corticosteroid treatment for the past three years with doses $>5 \mathrm{mg} /$ day; (5) not currently taking or previously taken a HMGCoA reductase inhibitors (statins) for the past 3 years. The same criteria apply to the elderly group except they may have concomitant osteoarthritis due to the high prevalence of this disease in the elderly population, which can be impossible to exclude.
The elderly participants were invited from a database of a collaborative research study [the Community Ageing Research 75+ (CARE 75+)], which provided the contacts of community-dwelling elderly volunteers aged above 75 who agreed to be approached by other research studies.

To select a healthy elderly cohort, all elderly participants had an Edmonton frailty scale of $\leq 5$ (not frail), an English Longitudinal Study of Aging (ELSA) frailty index score of $\leq 14$ ('fit' and 'well') and Montreal Cognitive Assessment (MoCA) score of $\geq 20$ (no signs of dementia). Moreover, they were screened to ensure none were sarcopenic according to the European working group on sarcopenia in older people (EWGSOP) criteria [1]. Muscle mass was measured using the Tanita DC-430 MA (Tanita Europe B.V., UK) bioelectrical impedance analysis system. The sarcopenia assessment flowchart is illustrated in Supplementary Fig. 1 highlighting that all elderly subjects were non-sarcopenic.

\section{Shear wave elastography}

SWE was performed using the two-dimensional Aixplorer (Supersonic Imagine, Aix-en-Provence, France) system using the SuperLinear ${ }^{\mathrm{TM}}$ SL10-2 MHz probe. The principle of SWE can be found in other reviews [5]. Briefly, the system evaluates tissue stiffness by measuring the propagation velocity of shear waves inside the tissue in meters per second $(\mathrm{m} / \mathrm{s})$. The shear wave velocity (SWV) increases proportionally with the elasticity modulus and can be used as a surrogate for tissue stiffness.

The technical acquisition methods were adapted from our previous work $[12,13]$. Namely, the ultrasound probe was oriented along the muscle fibres. The region of interest (ROI) size was set at $10 \mathrm{~mm}$. SWE acquisitions were repeated three times per muscle and recorded in units of meters per second $(\mathrm{m} / \mathrm{s})$. The probe was placed on top of the skin with a minimal load ensuring no external pressure could affect the measurements.

The muscles investigated were the four quadriceps [vastus lateralis (VL), rectus femoris (RF), vastus medialis (VM) and vastus intermedius (VI)], the three hamstrings [biceps femoris (BF), semitendinosus (ST) and semimembranosus (SM)] and the biceps brachii (BB). The muscles were scanned in the most relaxed muscle positions (Fig. 1). The quadriceps were also assessed during static passive stretching $\left(90^{\circ}\right.$ knee flexion position) to investigate the muscle elastic property when the muscle fibres are elongated under passive tension without any active force or load applied. The other muscles were not tested during static passive stretching to avoid prolonging the total scanning time (30 $\mathrm{min})$. These positions demonstrated reliable readings in our previous work and other studies $[12,13]$. The scan was limited to the dominant side since limb dominance had no significant impact on muscle stiffness [12]. Before scanning, all 


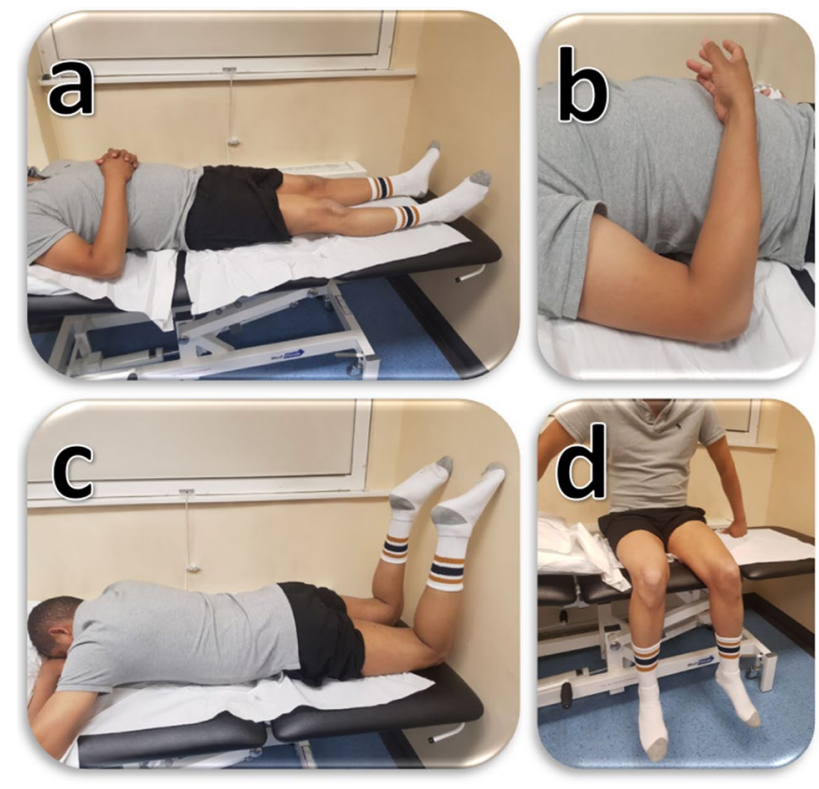

Fig. 1 Muscle SWE measurement positions. a (quadriceps rested): supine on a flat bed. b (biceps brachii rested): elbow flexed at $90^{\circ}$ with the forearm rested on the body and hand in supination. $\mathbf{c}$ (hamstrings rested): prone on a flat bed with the knees flexed at $90^{\circ}$ and rested on a wall. d (quadriceps passively stretched); seated with the hips and knees flexed at $90^{\circ}$ and feet hanging outside the table without touching the floor

participants were placed in a supine position on the scanning bed and were asked to relax and be comfortable for $5 \mathrm{~min}$. All participants were asked to refrain from any strenuous or sporting activities at least 1 day prior to the study to minimise possible confounding exercise effect.

\section{Muscle assessments}

\section{Handgrip strength}

Isometric handgrip muscle strength was tested using the Jamar Plus $+{ }^{\circledR}$ electronic hand dynamometer (Lafayette Instrument Company, USA). The participants were asked to perform maximal voluntary contraction, and the mean strength of three measurements was recorded for the dominant hand.

\section{Expanded timed get-up-and-go (ETGUG)}

Participants were asked to stand from a chair (sit to stand) then walk at their normal pace towards a cone placed 9.5 metres away (gait initiation and walk 1), turn around and walk back towards the chair (turn around and walk 2) then sit down (slow, stop, turn and sit). The times to perform these tasks were recorded using a stopwatch.

\section{0-s chair stand test (CST)}

Participants were asked to sit in the middle of a standard $43 \mathrm{~cm}$ high chair placed against a wall with their arms crossed over chest, feet flat on floor and shoulder width apart before being instructed to perform the maximum number of chair sit to stands they can within 30-s. The total number of sit to stands within the 30-s window was recorded.

\section{Knee extension/flexion strength}

The Biodex system 4 (IRPS Mediquipe, UK) was used to assess the isokinetic knee extension and flexion strengths. The chosen protocol tested the concentric isokinetic knee extension and flexion movements set at $60 \%$ s angular velocity. The range of motion was set at the maximum that each participant can achieve to obtain maximal speed at the isokinetic motion (usually ranged from $0^{\circ}-90^{\circ}$ to $0^{\circ}-120^{\circ}$ ). The participants performed three sets of three knee extension and flexion repetitions in sequence at $100 \%$ effort. They had a 30 -s rest period between the sets. The mean extension and flexion weight-normalised peak torque [Newton-meters $(\mathrm{Nm} / \mathrm{kg})]$ and average power (Watts $/ \mathrm{kg}$ ) were recorded.

\section{Statistical analysis methodology}

The $G^{*}$ Power statistical power analyses software was used to calculate the sample size for the one-way ANOVA test based on the effect size from a relatively similar previous study [14]. At alpha $=0.05$, power $(1-$ Beta $)=0.90$, effect size of 0.48 between the three groups, the required sample size is $n=60$, suggesting at least 20 participants per group.

Statistical analyses were undertaken using SPSS version 25 (Armonk, NY: IBM Corp). The one-way ANOVA test was used to determine if there is a statistically significant difference in SWV amongst the various age groups with post-hoc Tukey-corrected pairwise multiple comparisons to highlight differences between each group and another.

The association between SWV and the muscle assessment variables was tested using Pearson's correlation coefficients to understand how muscle stiffness correlates with strength and clinical characteristics. Moreover, the correlations with elderly-specific variables (ELSA frailly index, the SF-36 physical function and SF-36 general health) were evaluated. To further understand the underlying muscle stiffness process, multiple linear regression analysis was performed to evaluate if the independent variables (age, sex and BMI) are significantly associated with SWV. 


\section{Results}

\section{Participant information and muscle assessments}

A total of 77 participants volunteered in this study, of which 26 young, 21 middle-aged and 30 elderly with a mean age (SD, range) of 28.1 (4.1, 20-35), 48.5 (5.2, $40-55)$ and $81.7(4.1,77-94)$, respectively. The descriptive statistics of the main characteristics are listed in Table 1. The elderly group had a mean (SD) frailty index, SF-36 physical function and SF-36 general health scores of 9.5 (3.3), 71.4 (21.9) and 70.0 (13.4), respectively. Muscle mass decreased on average by $13.1 \%$ in the elderly group compared to young.

The one-way ANOVA results of the muscle assessments in Table 2 showed a significant difference amongst the three age groups $(p<0.05)$. Further analysis via post hoc multiple comparisons revealed that the significant differences only existed between the elderly group and the two younger groups. However, the knee extension torque and power were the only tests that showed significant differences between all groups $(p<0.001)$. The results for the subcomponents of the ETGUG test were similar to each other; hence, only the total ETGUG test time was reported. The elderly group were approximately $4 \mathrm{~s}$ slower in the ETGUG test. They had a significantly lower grip strength of $26.3 \mathrm{~kg}$ compared to $36.7 \mathrm{~kg}$ and $35.4 \mathrm{~kg}$ for young and middle-aged, respectively. On average, the young and middle-aged performed 5-7 chair stands in $30 \mathrm{~s}$ more than the elderly $(p<0.001)$.

\section{Shear wave elastography}

The SWE measurements listed in Table 3 identified significant differences $(p<0.001)$ during the resting position for
Table 1 Main characteristics of the study participants

\begin{tabular}{llllr}
\hline Characteristic & Young $(n=26)$ & Middle-aged $(n=21)$ & Elderly $(n=30)$ & $p$ value $^{\ddagger}$ \\
\hline Sex & 13 Females $(50 \%)$ & 15 Females $(71 \%)$ & 13 Females $(56 \%)$ & 0.32 \\
Age (years) & $28.1(4.1)$ & $48.5(5.2)$ & $81.7(4.1)$ & $<\mathbf{0 . 0 0 1}$ \\
Males (years) & $28.0(4.0)$ & $47.1(5.2)$ & $82.0(4.2)$ & $<\mathbf{0 . 0 0 1}$ \\
Females (years) & $28.3(4.3)$ & $49.1(5.3)$ & $81.4(4.1)$ & $<\mathbf{0 . 0 0 1}$ \\
Height (cm) & $169.0(10.6)$ & $167.2(10.2)$ & $161.2(7.3)$ & $\mathbf{0 . 0 0 7}$ \\
Weight (kg) & $71.3(21.9)$ & $71.7(12.2)$ & $75.5(12.6)$ & 0.57 \\
BMI & $24.5(5.3)$ & $25.6(3.6)$ & $28.8(4.7)$ & $\mathbf{0 . 0 0 2}$ \\
Fat mass (kg) & $16.1(10.6)$ & $18.9(5.0)$ & $26.7(8.6)$ & $<\mathbf{0 . 0 0 1}$ \\
Muscle mass (kg) & $53.3(14.8)$ & $51.0(10.7)$ & $46.3(8.2)$ & 0.08 \\
Muscle mass index & $18.3(3.1)$ & $17.7(2.2)$ & $17.7(2.3)$ & 0.69 \\
Smoking & $5(23 \%)$ & $7(33 \%)$ & $11(37 \%)$ & 0.18 \\
Pack-years & $1.25(4.3)$ & $1.8(1.3)$ & $7.5(16.5)$ & 0.16 \\
Drinking alcohol & $13(50 \%)$ & $10(43 \%)$ & $21(70 \%)$ & 0.12 \\
units/week) $^{\mathrm{a}}$ & $5.0(13)$ & $6.0(6.5)$ & $5.0(12)$ & 0.58 \\
VAS score (mm) $^{\mathrm{a}}$ & $6.0(14)$ & $6.5(17)$ & $10.0(25)$ & 0.25 \\
\hline
\end{tabular}

${ }^{\mathrm{a}}$ Median and interquartile range

${ }^{\ddagger} p$ values significant at $95 \%$ are highlighted in bold

Table 2 Results of the muscle assessments for the three age groups

\begin{tabular}{|c|c|c|c|c|c|c|c|c|}
\hline \multirow[t]{2}{*}{ Characteristic } & \multicolumn{2}{|l|}{ Young $^{(a)}$} & \multicolumn{2}{|c|}{ Middle aged $^{(\mathrm{b})}$} & \multicolumn{2}{|l|}{ Elderly $^{(\mathrm{c})}$} & \multirow[t]{2}{*}{$p$ value } & \multirow[t]{2}{*}{ Post hoc ${ }^{\mathrm{a}}$} \\
\hline & Mean (SD) & $95 \% \mathrm{CI}$ & Mean (SD) & $95 \% \mathrm{CI}$ & Mean $^{\mathrm{a}}$ & $95 \% \mathrm{CI}$ & & \\
\hline ETGUGT, total time-(s) & $16.1(1.4)$ & $15.6-16.7$ & $16.4(3.1)$ & $15.0-17.8$ & $20.6(3.6)$ & $19.3-22.0$ & $<0.001$ & $a, b<c$ \\
\hline 30-s chair sit-to-stands & $20.0(5.8)$ & $17.7-22.4$ & $17.9(3.1)$ & $15.3-20.6$ & $12.3(4.4)$ & $10.7-14.0$ & $<0.001$ & $\mathrm{a}, \mathrm{b}<\mathrm{c}$ \\
\hline Handgrip strength-(kg) & $36.7(10.8)$ & $32.3-41.2$ & $35.4(9.0)$ & $31.3-39.5$ & $26.3(10.6)$ & $22.4-30.3$ & 0.001 & $\mathrm{a}, \mathrm{b}<\mathrm{c}$ \\
\hline Knee extension torque-( $\mathrm{Nm} / \mathrm{kg})$ & $1.9(0.6)$ & $1.7-2.2$ & $1.5(0.5)$ & $1.2-1.7$ & $0.8(0.3)$ & $0.7-0.9$ & $<0.001$ & $a>b>c$ \\
\hline Knee flexion torque-(Nm/kg) & $1.0(0.3)$ & $0.9-1.1$ & $0.9(0.2)$ & $0.7-1.0$ & $0.5(0.2)$ & $0.4-0.5$ & $<0.001$ & $a, b>c$ \\
\hline Knee extension power-(W/kg) & $1.2(0.4)$ & $1.0-1.4$ & $0.9(0.3)$ & $0.7-1.0$ & $0.4(0.2)$ & $0.4-0.5$ & $<0.001$ & $a>b>c$ \\
\hline Knee flexion power-(W/kg) & $0.6(0.2)$ & $0.5-0.7$ & $0.5(0.1)$ & $0.4-0.6$ & $0.3(0.1)$ & $0.2-0.3$ & $<0.001$ & $a, b>c$ \\
\hline
\end{tabular}

${ }^{a}$ Result of the post hoc Tukey-corrected multiple comparisons 
Table 3 Muscle shear wave velocity in the healthy young, middle-aged and elderly participants

\begin{tabular}{|c|c|c|c|c|c|c|c|c|}
\hline \multirow[t]{2}{*}{ Muscle } & \multicolumn{2}{|l|}{ Young $^{(a)}$} & \multicolumn{2}{|c|}{ Middle aged $^{(b)}$} & \multicolumn{2}{|l|}{ Elderly $^{(\mathrm{c})}$} & \multirow[t]{2}{*}{$p$ value ${ }^{*}$} & \multirow[t]{2}{*}{ Post hoc ${ }^{\mathrm{a}}$} \\
\hline & Mean (SD) & $95 \%$ CI & Mean (SD) & $95 \%$ CI & Mean (SD) & $95 \% \mathrm{CI}$ & & \\
\hline Vastus lateralis-(VL) & $1.77(0.15)$ & $1.70-1.83$ & $1.64(0.12)$ & $1.59,1.70$ & $1.48(0.16)$ & $1.42-1.53$ & $<0.001$ & $a>b>c$ \\
\hline Passively stretched & $2.77(0.24)$ & $2.68-2.87$ & $2.72(0.42)$ & $2.53,2.92$ & $2.68(0.31)$ & $2.56-2.80$ & 0.54 & - \\
\hline Rectus femoris-(RF) & $1.80(0.14)$ & $1.74-1.85$ & $1.72(0.11)$ & $1.67,1.77$ & $1.58(0.16)$ & $1.52-1.64$ & $<0.001$ & $a, b>c$ \\
\hline Passively stretched & $2.21(0.21)$ & $2.13-2.30$ & $2.07(0.18)$ & $1.99,2.16$ & $2.25(0.41)$ & $2.09-2.40$ & 0.18 & - \\
\hline Vastus Medialis-(VM) & $1.71(0.12)$ & $1.67-1.76$ & $1.68(0.14)$ & $1.62,1.74$ & $1.38(0.10)$ & $1.34-1.42$ & $<0.001$ & $a, b>c$ \\
\hline Passively stretched & $2.56(0.20)$ & $2.48-2.64$ & $2.50(0.26)$ & $2.38,2.62$ & $2.45(0.28)$ & $2.34-2.55$ & 0.21 & - \\
\hline Vastus Intermedius-(VI) & $1.91(0.11)$ & $1.86-1.96$ & $1.92(0.19)$ & $1.83,2.01$ & $1.70(0.22)$ & $1.61-1.78$ & $<0.001$ & $a, b>c$ \\
\hline Passively stretched & $2.46(0.28)$ & $2.34-2.58$ & $2.39(0.33)$ & $2.24,2.54$ & $2.44(0.40)$ & $2.28-2.59$ & 0.72 & - \\
\hline Biceps Brachii-(BB) & $1.95(0.22)$ & $1.87-2.04$ & $1.85(0.15)$ & $1.78,1.92$ & $1.73(0.18)$ & $1.67-1.80$ & $<0.001$ & $a, b>c$ \\
\hline Biceps Femoris-(BF) & $1.73(0.12)$ & $1.68-1.78$ & $1.64(0.14)$ & $1.58,1.70$ & $1.40(0.17)$ & $1.34-1.47$ & $<0.001$ & $a, b>c$ \\
\hline Semitendinosus-(ST) & $1.71(0.10)$ & $1.67-1.75$ & $1.64(0.14)$ & $1.57,1.70$ & $1.38(0.16)$ & $1.32-1.44$ & $<0.001$ & $a, b>c$ \\
\hline Semimembranosus-(SM) & $1.80(0.12)$ & $1.75-1.85$ & $1.70(0.12)$ & $1.65,1.76$ & $1.38(0.17)$ & $1.32-1.45$ & $<\mathbf{0 . 0 0 1}$ & $a, b>c$ \\
\hline
\end{tabular}

*Significant one-way ANOVA $p$ values at $95 \%$ are highlighted in bold

${ }^{a}$ Result of the post hoc Tukey-corrected multiple comparisons

all the tested lower limb muscles and the BB. The SWV was higher in the passively stretched position in the quadriceps. However, it did not result in significant differences amongst the age groups. The results can be better appreciated graphically in the clustered error bars in Fig. 2 during the resting position and in Fig. 3 during the passive stretching position.

The mean SWV difference between the middle-aged and young groups over all muscles was $-4.1 \%$ ranging from 0.5 to $-7.3 \%$. As for the elderly and middle-aged, the mean difference was $-12.9 \%$ and ranged from -6.5 to $-18.8 \%$. However, the highest mean difference of $-16.5 \%$ was observed between the elderly and young groups with the differences amongst the muscles ranging from -11.0 to $-23.3 \%$. The VI and BB exhibited the smallest differences, whereas the VM and SM were the highest.
Despite the gradual decreasing SWV across almost all muscle, the post hoc analysis revealed that only the VL had a significantly decreasing SWV between all three age groups. The other muscles were only significantly lower in the elderly participants $(p<0.001)$ compared to the other two groups. SWE examples are shown in Fig. 4.

The correlations between SWV and the main clinical and muscle assessment variables are listed in Table 4. On average, there were significant moderate correlations between SWV and the tested variables. Age had a significant negative correlation ranging from -0.450 to -0.822 for the BB and SM, respectively $(p<0.001)$. The muscle mass index, however, did not correlate with any of the muscle SWV $(r<0.15 ; p>0.05)$. The strongest correlations were found for the Biodex knee extension/flexion
Fig. 2 Clustered error bars for the mean muscle shear wave velocity in the relaxed resting position. The asterisks indicate significance at 0.05 level $(*)$ and $0.01(* *)$. The hyphens $(-)$ indicate lack of statistical significance $(p>0.05)$

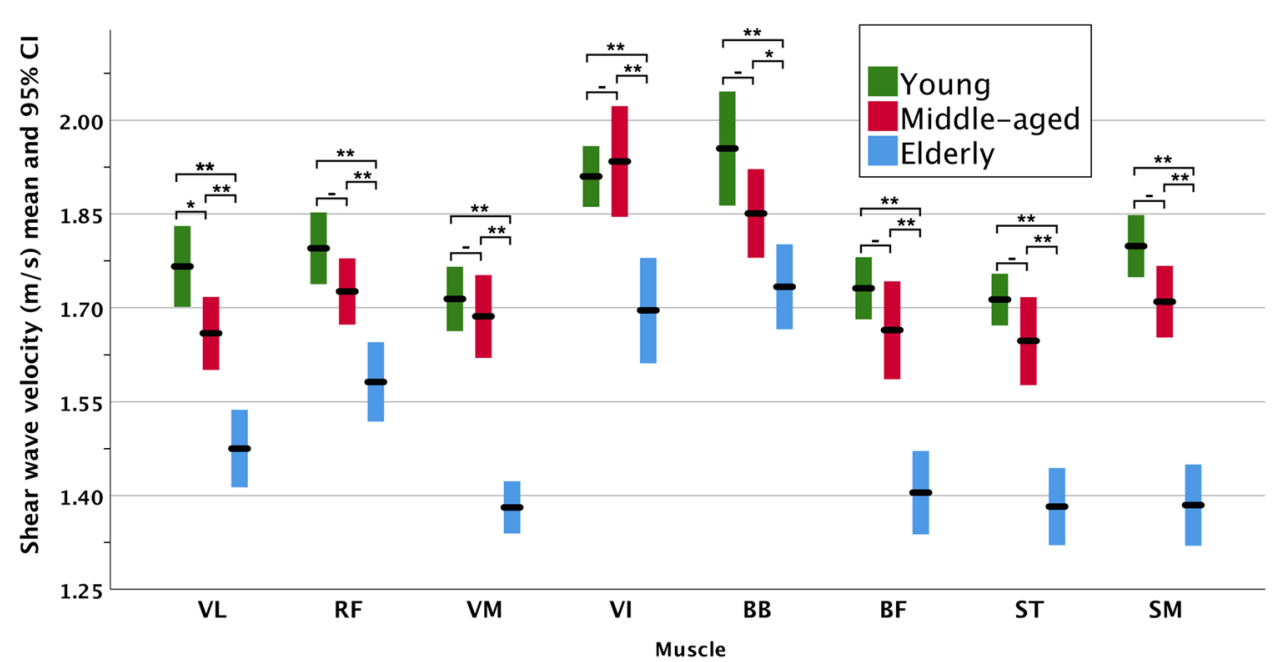


Fig. 3 Clustered error bars for the mean muscle shear wave velocity during in the passively stretched position. The hyphens (-) above the bars indicate lack of statistical significance $(p>0.05)$

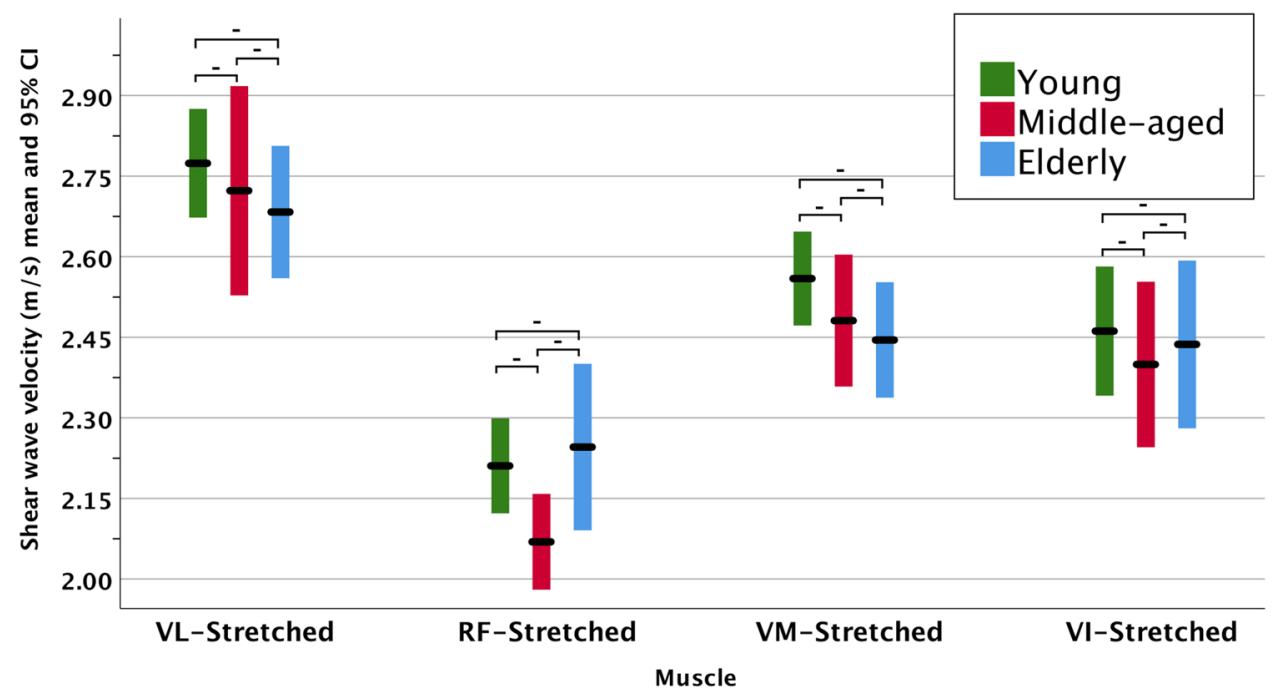

However, there were no significant correlations with muscle mass or muscle mass index.

The multiple linear regression determined that neither sex or BMI explained a significant variation in SWV in all muscles. In contrast, age was consistently a significant predictor of SWV in the regression model. The adjusted $R^{2}$ for the VL, RF, VM, VI, BB, BF, ST and SM were 0.455, 0.314, $0.587,0.205,0.191,0.547,0.600$ and 0.671 , respectively. In other words, $45.5 \%$ of the variance in SWV is explained by age in the VL muscle as an example. The results of the two models on the investigated muscles are included in Supplementary Table 1.

\section{Discussion}

The primary aim of this study was to investigate the effect of ageing on muscle stiffness using SWE. To our knowledge, this is the first study to incorporate strength and functional correlations to substantiate their associations with muscle stiffness throughout an adult age-span. We systematically scanned all quadriceps and hamstring muscles, recommended in a previous study and not been simultaneously investigated before [14]. Additionally, the majority of prior work have not included elderly subjects above 75 , which has previously been recommended [15]. We achieved this by recruiting healthy elderly participants up to 94 years.

The results demonstrated a gradual reduction in resting muscle stiffness throughout adulthood with a significant decline most notable in the elderly ( $>75$ years) group. This was true for all the tested muscles, which display different fibre arrangements and functions. These findings support the hypothesis that known age-related histological features of muscle such as myosteatosis, variability in myofiber size and impaired connective tissue may promote the loss of normal 
Table 4 Muscle shear wave velocity correlations with clinical and muscle test variables for all participants

\begin{tabular}{lllllllll}
\hline & VL & RF & VM & VI & BF & ST & SM & BB \\
\hline Age & $-0.680^{\mathrm{b}}$ & $-0.568^{\mathrm{b}}$ & $-0.770^{\mathrm{b}}$ & $-0.464^{\mathrm{b}}$ & $-0.744^{\mathrm{b}}$ & $-0.778^{\mathrm{b}}$ & $-0.822^{\mathrm{b}}$ & $-0.450^{\mathrm{b}}$ \\
BMI & $-0.346^{\mathrm{b}}$ & $-0.323^{\mathrm{b}}$ & -0.210 & $-0.323^{\mathrm{b}}$ & $-0.315^{\mathrm{b}}$ & $-0.316^{\mathrm{b}}$ & $-0.427^{\mathrm{b}}$ & $-0.250^{\mathrm{a}}$ \\
Fat mass & $-0.453^{\mathrm{b}}$ & $-0.421^{\mathrm{b}}$ & $-0.385^{\mathrm{b}}$ & $-0.392^{\mathrm{b}}$ & $-0.474^{\mathrm{b}}$ & $-0.414^{\mathrm{b}}$ & $-0.542^{\mathrm{b}}$ & $-0.330^{\mathrm{b}}$ \\
Muscle mass & 0.216 & 0.146 & $0.276^{\mathrm{a}}$ & 0.226 & $0.266^{\mathrm{a}}$ & 0.231 & $0.316^{\mathrm{b}}$ & 0.189 \\
ETGUGT, total time & $-0.386^{\mathrm{b}}$ & $-0.288^{\mathrm{a}}$ & $-0.367^{\mathrm{b}}$ & $-0.380^{\mathrm{b}}$ & $-0.338^{\mathrm{b}}$ & $-0.366^{\mathrm{b}}$ & $-0.560^{\mathrm{b}}$ & -0.198 \\
30-s Chair stand test & $0.354^{\mathrm{b}}$ & $0.294^{\mathrm{b}}$ & $0.482^{\mathrm{b}}$ & $0.261^{\mathrm{a}}$ & $0.373^{\mathrm{b}}$ & $0.365^{\mathrm{b}}$ & $0.522^{\mathrm{b}}$ & $0.267^{\mathrm{a}}$ \\
Handgrip Strength & $0.285^{\mathrm{a}}$ & $0.256^{\mathrm{a}}$ & $0.343^{\mathrm{b}}$ & $0.293^{\mathrm{a}}$ & $0.344^{\mathrm{b}}$ & $0.297^{\mathrm{b}}$ & $0.436^{\mathrm{b}}$ & $0.287^{\mathrm{a}}$ \\
Knee extension torque & $0.587^{\mathrm{b}}$ & $0.454^{\mathrm{b}}$ & $0.636^{\mathrm{b}}$ & $0.356^{\mathrm{b}}$ & $0.547^{\mathrm{b}}$ & $0.542^{\mathrm{b}}$ & $0.640^{\mathrm{b}}$ & $0.443^{\mathrm{b}}$ \\
Knee flexion torque & $0.540^{\mathrm{b}}$ & $0.405^{\mathrm{b}}$ & $0.603^{\mathrm{b}}$ & $0.385^{\mathrm{b}}$ & $0.497^{\mathrm{b}}$ & $0.491^{\mathrm{b}}$ & $0.641^{\mathrm{b}}$ & $0.422^{\mathrm{b}}$ \\
Knee flexion power & $0.579^{\mathrm{b}}$ & $0.447^{\mathrm{b}}$ & $0.613^{\mathrm{b}}$ & $0.350^{\mathrm{b}}$ & $0.535^{\mathrm{b}}$ & $0.501^{\mathrm{b}}$ & $0.623^{\mathrm{b}}$ & $0.456^{\mathrm{b}}$ \\
Knee extension power & $0.540^{\mathrm{b}}$ & $0.439^{\mathrm{b}}$ & $0.600^{\mathrm{b}}$ & $0.333^{\mathrm{b}}$ & $0.489^{\mathrm{b}}$ & $0.479^{\mathrm{b}}$ & $0.640^{\mathrm{b}}$ & $0.422^{\mathrm{b}}$ \\
\hline
\end{tabular}

${ }^{a}$ Correlation is significant at the 0.05 level (2-tailed)

${ }^{\mathrm{b}}$ Correlation is significant at the 0.01 level (2-tailed) muscle stiffness. This was also proposed previously by the work of Rodrigues \& Rodrigues Junior [9], consistent with the elastic fibre system in the muscle extracellular matrix starting to lose its resistance property and becoming softer with ageing.

In contrast, this study has not confirmed previous in-vitro results by Ochala et al. [16], which reported increased muscle stiffness with ageing due to increased collagen concentrations. Brown et al. [7], in contrast, reported that muscle stiffness is not significantly different between young and old rats. However, these studies used a fundamentally different biomechanical approach (testing the passive tension-length relationship) for evaluating muscle stiffness. There is also a lack of consensus in the SWE literature as to whether muscle stiffness decreases [14, 17], increases [18] or does not change [19] with ageing. Akagi et al. [14] reported a significant reduction of $17 \%$ in the RF stiffness, decreasing from $3.4 \mathrm{kPa}$ in the young to $2.8 \mathrm{kPa}$ in the elderly $(p=<0.001)$. Similarly, Yoshida et al. [17] found a comparable decline in muscle stiffness of approximately $9 \%$ in the gastrocnemius. These percentages are similar to the observed average range of reduction in this study.

Studies using SWE [19] and magnetic resonance elastography [20] confirm the observed decreasing trend with a lack of statistical significance between young and middleaged groups. The muscle ageing process does not appear to start until the sixth decade when declines in muscle strength and mass emerge [21]. Significant declines take place above 75 years as risks of fall and frailty increase [22]. This probably explains the overall lack of difference between young and middle-aged, except in VL.

It is interesting that muscle stiffness during passive stretching is preserved in older age, which was also observed in a previous study on the VI muscle [19]. The mechanism behind this remains unclear. Considering that all of the recruited participants were healthy, it may be speculated that passive muscle stiffness is preserved to maintain normal muscle contractility and function.

The secondary aim of this study was to elucidate the relationship of muscle stiffness to muscle mass and strength, the principal components of sarcopenia and dynapenia, respectively. There was a stronger correlation with fat mass compared to muscle mass, which correlated poorly with just two of the scanned muscles. On the other hand, weaker muscles and worse physical performance were associated with decreased muscle stiffness. Considering that the increase in muscle compliance is related to a decrease in muscle strength [23], the observed loss in muscle stiffness in the elderly group may explain the positive correlation with low muscle strength.

The implications of this study findings are important. The decreased muscle stiffness in the elderly subject and its correlation with reduced strength could compromise the delivery of tensile muscle power of the muscle fibres to the tendon. This may suggest an increased exposure risk of muscle fibres to rupture or deformation, consequently increasing rates of physical frailty. Hence, SWE could be a useful tool for evaluating and screening elderly subjects at risk of sarcopenia and frailty. Besides, the higher average reduction between elderly and young in muscle stiffness (16.5\%) compared to muscle mass (13.1\%) may speculate a better role of SWE in predicting earlier muscle changes associated with ageing. However, further research is required to establish these roles. In terms of future study design, the results highlight the importance of adjusting for age in future muscle SWE studies, especially when the cohort involves a wide age range.

The results hold promise for SWE in the field of elderly care medicine for highlighting the impact of ageing on muscle stiffness. Future studies should aim to compare muscle stiffness in sarcopenic and non-sarcopenic subjects to elucidate the value of SWE for assessing muscle quality. 
Currently, abnormal muscle stiffness is not a risk factor for falls in the elderly population; researching this could yield valuable evidence. Future studies are also encouraged to control for physical activity to strengthen the relationship between muscle stiffness and age.

The findings in this study are subject to several limitations. First, the three age groups were not matched in size. Second, the study did not evaluate the passive stretching on the $\mathrm{BB}$ or hamstring muscle to avoid long scanning time. Moreover, the study design does not represent the complete adulthood age-span since it lacked information on younger subjects 56-74 years. Finally, inter-operator reproducibility was not verified.

In conclusion, ageing was associated with a decline in both lower and upper limb skeletal resting muscle stiffness as measured by SWE. The greatest reduction in muscle stiffness was found in elderly participants $>75$ years. The decline correlated stronger with lower muscle strength than muscle mass. Sex and BMI did not influence muscle stiffness. The age-related changes in muscle stiffness may contribute to our understanding of the development of musculoskeletal disorders with age. The results highlight the needs for further research to investigate if decreased muscle stiffness can predict fall risk in elderly individuals. Overall, the results emphasise the importance of controlling for the age variable in muscle SWE studies.

Acknowledgements The research is supported by the National Institute for Health Research (NIHR) infrastructure at Leeds. The views expressed are those of the authors and not necessarily those of the NHS, the NIHR or the Department of Health. Some of the data in this study were made available through the Community Ageing Research $75+$ (CARE 75+), and we would like to thank them for their support in providing the information required to recruit the elderly participants. CARE75+ (ISRCTN16588124) is led by the Academic Unit of Elderly Care \& Rehabilitation based at the Bradford Teaching Hospitals NHS Foundation Trust, Bradford Teaching Hospitals NHS Foundation Trust.

\section{Compliance with ethical standards}

Conflict of interest All authors declare no potential conflict of interest.

Statement of human rights The design of the study complied with the Code of Ethics of the World Medical Association (Declaration of Helsinki,1964). Ethical approval was granted by the UK research ethics committee (Ref:17/EM/0079).

Informed consent All participants provided a written informed consent.

Open Access This article is distributed under the terms of the Creative Commons Attribution 4.0 International License (http://creativeco mmons.org/licenses/by/4.0/), which permits unrestricted use, distribution, and reproduction in any medium, provided you give appropriate credit to the original author(s) and the source, provide a link to the Creative Commons license, and indicate if changes were made.

\section{References}

1. Cruz-Jentoft AJ, Baeyens JP, Bauer JM et al (2010) Sarcopenia: European consensus on definition and diagnosis Report of the European Working Group on Sarcopenia in Older People. Age Ageing 39:412-423. https://doi.org/10.1093/ageing/afq034

2. Fukagawa NK, Wolfson L, Judge J et al (1995) Strength is a major factor in balance, gait, and the occurrence of falls. J Gerontol Ser A Biol Sci Med Sci 50(Special_Issue):64-67

3. Faulkner JA, Larkin LM, Claflin DR et al (2007) Age-related changes in the structure and function of skeletal muscles. Clin Exp Pharmacol Physiol 34:1091-1096. https://doi.org/10.111 1/j.1440-1681.2007.04752.x

4. Kragstrup TW, Kjaer M, Mackey A (2011) Structural, biochemical, cellular, and functional changes in skeletal muscle extracellular matrix with aging. Scand J Med Sci Sports 21:749-757

5. Sigrist RMS, Liau J, Kaffas AE et al (2017) Ultrasound elastography: review of techniques and clinical applications. Theranostics 7:1303-1329. https://doi.org/10.7150/thno.18650

6. Hsiao M-Y, Chen Y-C, Lin C-Y et al (2015) Reduced Patellar tendon elasticity with aging: in vivo assessment by shear wave elastography. Ultrasound Med Biol 41:2899-2905. https://doi. org/10.1016/j.ultrasmedbio.2015.07.008

7. Brown M, Fisher JS, Salsich G (1999) Stiffness and muscle function with age and reduced muscle use. J Orthop Res 17:409-414. https://doi.org/10.1002/jor.1100170317

8. Gao Y, Kostrominova TY, Faulkner JA et al (2008) Age-related changes in the mechanical properties of the epimysium in skeletal muscles of rats. J Biomechan 41:465-469

9. Rodrigues CJ, Rodrigues Junior AJ (2000) A comparative study of aging of the elastic fiber system of the diaphragm and the rectus abdominis muscles in rats. Braz J Med Biol Res 33:1449-1454

10. Vandervoort AA (2002) Aging of the human neuromuscular system. Muscle Nerve 25:17-25. https://doi.org/10.1002/mus.1215

11. Kragstrup TW, Kjaer M, Mackey AL (2011) Structural, biochemical, cellular, and functional changes in skeletal muscle extracellular matrix with aging. Scand J Med Sci Sports 21:749-757. https ://doi.org/10.1111/j.1600-0838.2011.01377.x

12. Alfuraih AM, O'Connor P, Hensor E et al (2018) The effect of unit, depth, and probe load on the reliability of muscle shear wave elastography: variables affecting reliability of SWE. J Clin Ultrasound 46:108-115. https://doi.org/10.1002/jcu.22534 doi

13. Alfuraih AM, O'Connor P, Tan AL et al (2017) An investigation into the variability between different shear wave elastography systems in muscle. Med Ultrasonogr 19:392-400. https://doi. org/10.11152/mu-1113

14. Akagi R, Yamashita Y, Ueyasu Y (2015) Age-related differences in muscle shear moduli in the lower extremity. Ultrasound Med Biol 41:2906-2912. https://doi.org/10.1016/j.ultrasmedb io.2015.07.011

15. Domire ZJ, McCullough MB, Chen Q et al (2009) Feasibility of using magnetic resonance elastography to study the effect of aging on shear modulus of skeletal muscle. J Appl Biomech 25:93-97

16. Ochala J, Frontera WR, Dorer DJ et al (2007) Single skeletal muscle fiber elastic and contractile characteristics in young and older men. J Gerontol Ser A Biol Sci Med Sci 62:375-381. https://doi. org/10.1093/gerona/62.4.375

17. Yoshida K, Itoigawa Y, Maruyama Y et al (2017) Application of shear wave elastography for the gastrocnemius medial head to tennis leg. Clin Anat 30:114-119

18. Eby SF, Cloud BA, Brandenburg JE et al (2015) Shear wave elastography of passive skeletal muscle stiffness: influences of sex and age throughout adulthood. Clin Biomech 30:22-27 
19. Wang CZ, Guo JY, Li TJ et al (2017) Age and sex effects on the active stiffness of vastus intermedius under isometric contraction. BioMed Res Int 2017:9469548. https://doi. org/10.1155/2017/9469548

20. Debernard L, Robert L, Charleux F et al (2011) Analysis of thigh muscle stiffness from childhood to adulthood using magnetic resonance elastography (MRE) technique. Clin Biomech 26:836-840. https://doi.org/10.1016/j.clinbiomech.2011.04.004

21. Silva AM, Shen W, Heo M et al (2010) Ethnicity-related skeletal muscle differences across the lifespan. Am J Hum Biol 22:76-82. https://doi.org/10.1002/ajhb.20956

22. Mitchell W, Atherton P, Williams J et al (2012) Sarcopenia, dynapenia, and the impact of advancing age on human skeletal muscle size and strength; a quantitative review. Front Physiol. https://doi.org/10.3389/fphys.2012.00260

23. Costa PB, Ryan ED, Herda TJ et al (2009) Acute effects of passive stretching on the electromechanical delay and evoked twitch properties. Eur J Appl Physiol 108:301. https://doi.org/10.1007/ s00421-009-1214-3

Publisher's Note Springer Nature remains neutral with regard to jurisdictional claims in published maps and institutional affiliations. 